\title{
A Multi Agent System to Manage Ideas during Collaborative Creativity Workshops
}

\author{
Davy Monticolo and Simona Mihaita
}

\begin{abstract}
During creativity workshops, participants and creativity quizmasters work together in a collaborative way to generate new ideas. They will follow the creativity process based on different phases: discover the topic, apply the creativity methods, generate ideas, formalize ideas, share and evaluate their ideas and select innovative ideas. Up to now, the creativity workshops use simple tools like paperboards, post-its or idea sheets to capture their ideas. With such tools they don't have the possibility to evaluate, compare, share and select their ideas. In this paper we describe the architecture of a multi agent system called CIMAS (Creativity Ideas Managed by Agent Systems) which helps the participants in a creativity workshop to generate, capture, collect and select their ideas.
\end{abstract}

Index Terms-Multi agent system, creativity, ideas ontology

\section{INTRODUCTION}

For many years scientists have been wondered if they can built new technical and innovative systems which would emulate a valuable human behavior consisting of visualizing and giving life to their ideas. This behavior is called creativity and involves many concepts such as: discovery, creation, sociability, refinement and communication.

There are more than 80 definitions of creativity in the literature [1]. The most popular is the one given by [2] which states that "creativity is the ability to generate new useful things that are characterized by being novel and appropriate".

Although many creativity works of arts are often seen as the creation of an individual, more complex and innovative results are obtained as a consequence of the collaboration and interaction between individuals [3]. Each individual's skills, originality, knowledge and expertise can easily built the road towards creative and sustainable solutions [4].

Scientific collaborations between computer scientists and domain specialists will significantly accelerate the development of a project compared to the work of a single artist/scientist [5], by using different creativity support tools. The design of today's modern computer-based environments will extend the user's capability to make discoveries and inventions from the early stages of the innovation process (by generating ideas, hypothesis and alternatives, gathering the information), through the later stages of validation, refinement and completion.

Although there is a large amount of literature in creativity and innovation, the topic is quite new in the field of computer and information science. The standing proof are the excellent

Manuscript received March 17, 2013; revised May 6, 2013.

The authors are with the ERPI laboratory of the University of Lorraine, Nancy, 54000 France (e-mail: davy.monticolo@ univ-lorraine.fr, imona.mihaita@univ-lorraine.fr). books on creativity ([6], [7], [8]) which unfortunately don't relate to the terms of "computer tools", "user interface", "interactive collaboration". In order to built innovative creativity support tools, we have to consider some principles that they should present [9] : support the exploratory search (search services which allow ranking, clustering, organizing and marking), enable collaboration in the community of social creativity, provide rich history-keeping, provide an easy design tool which can be easy to learn and apply for beginners.

Collaborative meetings often take place in co-located settings which are usually planned in advanced, but also in a spontaneous way. When working together and using traditional communicating methods (pen and paper, printed documents, reports, paperboard, etc.) around horizontal surfaces (tables, desks) people tend to better organize their ideas and thoughts [10]. But when moving to digital media (getting around a PC or working separately on their own laptop, listening to a video-conference, etc.) people loose the feeling of having a comfortable and a productive collaboration. Several research studies on how to choose a modern interactive tool (or even a collaboration ecology) which simulates the creativity collaborations have been recently presented in [11],[12], [13].

Creativity is the key to new successful ways of conducting a project, either it's in the educational field (professors looking for new teaching methods or student projects) or in the business domain (companies seeking for success through innovative products). Therefore companies and professional freelancers participate in the so-called creativity workshops, under the supervision of a creativity expert which leads the way though the workshop. For example the ArtScience Annual Innovation Workshop1 hosted by Le Laboratoire in Paris, France, gather students from all around the world towards highly innovative art and design ideas at frontiers of science. Another educational creativity workshop is the annual Creative Practice Bootcamp2 held in Nashville, TN, where students learn how to apply methods like Brainstorming [14], Brainpurge [15] or Brainwriting [16]. There are also a considerable number of companies which take part in the business creativity workshops, seeking new ideas for the development of their products, their marketing strategies, etc. One example is The Creativity Workshop3 held regularly in different cities around the world, or the Creative4Business Workshop 4 which aims at helping the participants to generate, filter and extract the most original

\footnotetext{
http://www.artsciencelabs.org/the-labs/

${ }^{2}$ http://curbcreativepracticebootcamp.eventbrite.com/

http://www.creativityworkshop.com/

${ }^{4}$ http://www.creative4business.co.uk/workshops/
} 
ideas. But all of the above mentioned workshops relate to the traditional pen-and-paper methods, not being able to process and to filter ideas automatically as the creativity process unfolds.

Recently, S. Buisine proposed in [17] some experiments of two creativity methods: Brainpurge and Minmapping [18]. They used an augmented multi-user tabletop system DiamonSpin Toolkit [19] on which they carried creative problem solving sessions; the same sessions were carried out using pens, paper, and flip charts [15]. Finally, subjective evaluation showed mixed results: users preferred pen-and-paper for the Brainpurge, but preferred the digital tabletop for the Mindmap.

The above studies lead us to propose a multi-agent system which is able to support the creativity process that we will detail in the next section.

\section{Multi Agent System to Support the Creativity PROCESS}

The population in a creativity workshop is by nature an heterogeneous and often distributed. Often, we observe three types of users in creativity workshop; the stakeholder who define the subject/problem to solve, the creativity quizmasters who animate the creativity sequences and the creative participants who create ideas by applying creativity methods. Furthermore, the creative participant groups are composed by heterogeneous people with different skills and responsibilities. In a creativity workshop, there are different participant groups which are working in the same time in different places.

The collaborative creative process is by nature an heterogeneous and distributed information landscape. As a matter of fact, the stakeholders will provide some information about the problem to solve. This information can be found under different formats like presentations, videos, photos or text. They will also answer to the creative participant groups by sending messages by direct talk. Otherwise the creative participants generate ideas by writing text, drawing sketches and can also present an idea with a simple post-it.

Multi-agents systems are a stage in abstraction that can be used to understand, to model and to develop a whole new class of distributed systems [20]. The MAS paradigm is well suited for designing software which is adapted to manage information in a heterogeneous and distributed information landscape [21]. On the one hand, individual agents locally adapt to the users and the resources they are dedicated to; on the other hand, cooperating agents enable the whole system to support a complex system like the management of ideas during a collaborative creativity workshop.

\section{OVERVIEW OF CIMAS}

In this section we describe the concepts and the architecture of the CIMAS system. There are three types of users; the stakeholders the creativity quizmasters and the creative participants. The aim objectives of the CIMAS system are:
- To help creative participant to annotate and evaluate their ideas and to research others similar ideas;

- To assist stakeholders to search relevant ideas by using different point of views;

- To assist creativity quizmaster by providing indicators and ideas trends from all the creative participant groups.

\section{A. Agents Dedicated to the Ideas Annotation}

Doyle explains in [22] that "annotated environments containing explanations of the purpose and uses of spaces and activities allow agents to quickly become intelligent actors in those spaces". The ideas landscape represents the annotated environment build by the agents. Indeed the CIMAS agents have to annotate each ideas, sketch or post-it in order to handle and exploit this information.

The web semantic [23] represents a set of languages which facilitate the annotation of web resources. By using RDF language of the web semantic, we can describe the context and the content of an idea even if the idea is a text, a sketch or a video. Compared to the Web, the ideas have more delimited context. We can easily define who the creators are, the type of content, when the idea was created. Thus an ontological approach is conceivable to describe ideas. Several ideas ontologies already exist like the "ideaontology" [24] and "Generic Idea Management ontology" [25]. Ideaontology is dedicated to the evaluation of the idea and use mono criteria methods to evaluate a idea. The second ontology is based on four groups of concepts; the concepts related to describe the origin of the idea, the concepts relative to describe the idea, the concepts which describe the innovative part of the idea (impact of the idea, target, feasibility, etc.), and the object (evolution of the idea, the process to develop it, etc.). In CIMAS we have build an ontology with only concepts related to describes the ideas (description, types, use cases, etc.) and to describe the context of a idea (creator, trust, evaluation, related project, etc.). The CIMAS ontology is formalized with OWL lite [26] which is related to provide a conceptual model to describe ideas and which the resources are defined separately.

The Fig. 1 shows an extract of the CIMAS ontology and an example of annotation with literal and conceptual properties.

The CIMAS system does not lead directly with the web resources but with their annotation to support the ideas information management. Thus the CIMAS ontology represents a conceptual structure used by the agents to annotate ideas, to organize and research them.

\section{B. Architecture}

A Multi Agent System is a network of agents that work together in a cooperative way to solve problems that would be generally difficult to solve for any individual agent. Information Agents are a part of intelligent agents [27], [28]. Klusch made a list of the services that a multi-agent system can offer in a information management approach [29]:

- Search, acquire, analyse and classify information coming from various information sources;

- Give information to human and computing networks once usable knowledge is ready to be consulted;

- Negotiate on information integration or exclusion into 
the system;

- Give explanation to the quality and reliability related to the integrated information;
- Learn progressively all along the information management process;

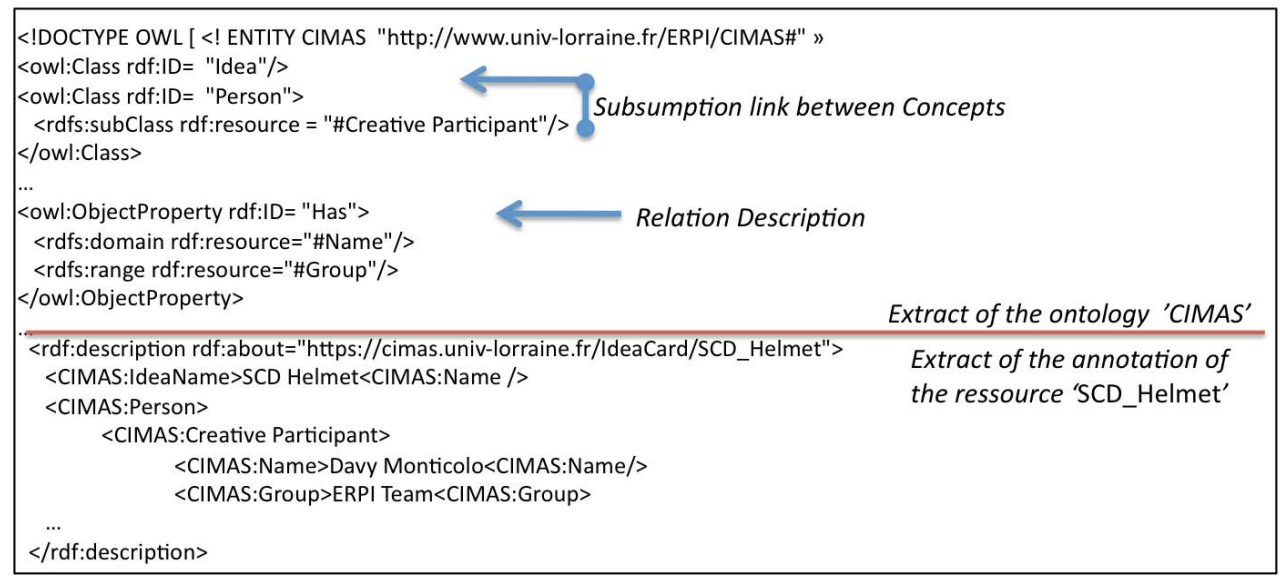

Fig. 1. Extract of the CIMAS ontology and annotation example.

The proposed approach to design a MAS is based on an organizational approach like the A.G.R model used in AALAADIN [30], OperettA [31] and methodologies like GAIA [20], TROPOS [32] or RIOCC [33]. Thus the CIMAS architecture is tackled as a human society in terms or role, skill and relationships.

The main objective of the CIMAS system is to manage ideas coming from different information sources (post-its, texts, sketches). The CIMAS system is based on three layers (Fig. 2):
- The ideas landscape where users insert their ideas (text, sketch or post-its) in the system by using forms;

- The agents layer where the management of ideas is executed;

- The Interface layer where users can research an idea, consult an idea card or display the ideas trend inside a creativity workshop.

In this paper we describe the agents layer.

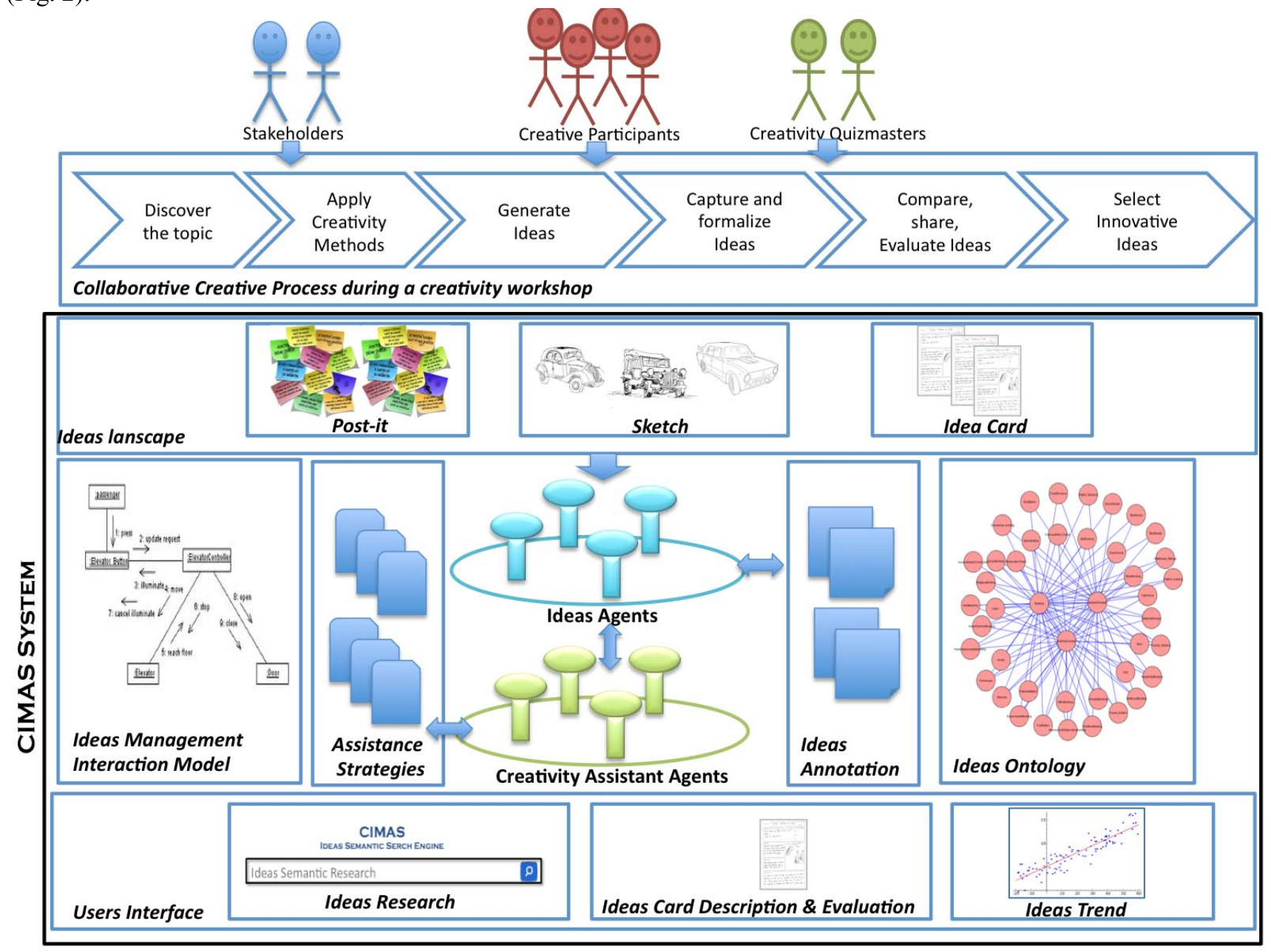

Fig. 2. CIMAS architecture 


\section{Ideas Agents Society in CIMAS}

From the architecture analysis we can specify the two different agents' societies, the interactions between agents and the mechanisms they use to handle ideas annotations.

The Ideas Society is dedicated to annotate the idea according to the ideas ontology. More explicitly, the agents use the structure of the ontology to annotate ideas. There are three Ideas agents, one by creativity workshop. There is one agent according to each type of content (post-it, sketch and idea card). The annotation of an idea is composed of a list of tags (Fig. 3), which describe its creation (creator, creation date, team, creativity workshop) and its evolution (Number of views, number of "likes", etc.).

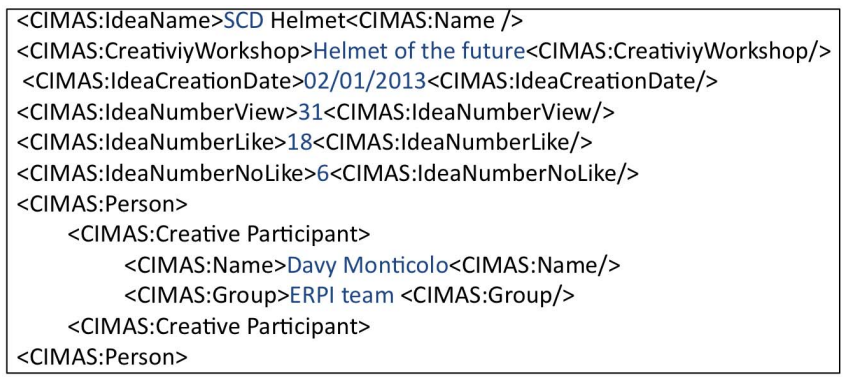

Fig. 3. Annotation of an idea

The Ideas agents also build the result of the semantic researches when users enter keywords in the CIMAS search engine (Figure 2). They use to mechanisms; the first one is to built SPARQL requests [34] and the second is to calculate the semantic similarity between two ideas.

The first method is used to research the exact name of an idea, a creator or a group. Figure 4 shows the SPARQL request to search an idea which is called "Unbreakable helmet" created by the group "ERPI team".

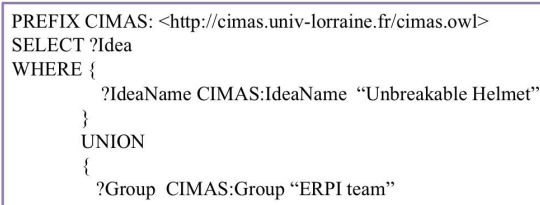

Fig. 4. Annotation of an idea

The second method consists of calculate the similarity between two concepts in order to identify the similar concepts or the closed concepts. The method is based on the calculus of the semantic distances between two concepts in the RDF models embedded annotations. For example in the CIMAS ontology we have the following property :

$$
[\text { Post }-i t] \rightarrow(\text { Creator }) \rightarrow[\text { CreativeParticipant }]
$$

The method will provide results such as:

$$
\begin{aligned}
& {[\text { Sketch }] \rightarrow(\text { Creator }) \rightarrow[\text { Student }]} \\
& {[\text { IdeaCard }] \rightarrow(\text { Creator }) \rightarrow[\text { Pr ofessor }]}
\end{aligned}
$$

To research the concepts which are close semantically the agents use the distance of Rada [35] counting the number of arcs on the shorter path between two terms ( $t 1$ and $t 2$ ) (formula 1). By using this distance we can define the distance between two RDF triplets as the sum of the distances between: two relations, two concepts in first argument (domain) and two concepts in second argument (range) (formula 2).

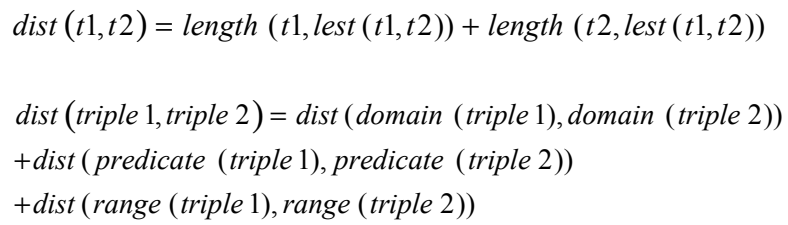

The algorithm gives a number between 0 and 1 . More the number is closed to 1; more the concepts are semantically close. A semantic research on all the RDF annotations on the word "Helmet" provides the results shown in Table I:

TABLE I: RESEARCH FOR THE WORD “HELMET” IN THE IDEAS ANNOTATIONS

\begin{tabular}{ll}
\hline Concepts & Similarity index \\
\hline Headdress & 0,632 \\
Hard Hat & 0,452 \\
Crash Helmet & 0,678 \\
Bandore & 0,128 \\
Crown & 0,321 \\
Hat Head Protector & 0,521 \\
Safety Helmet & 0,862 \\
\hline \hline
\end{tabular}

The Ideas agents will propose the three best results of the research.

\section{The Society of Creative Assistant Agents in CIMAS}

The Creativity Assistant Agents (CAA) interact with the users through the three following interfaces:

- The semantic research engine where they will send the elements of the request to the Ideas agent;

- The Ideas Card visualization and Evaluation. With this interface the CA presents the different idea cards and allow to the users to add a comment or a mention "like" or "not like";

- The Ideas Trend interface. This interface is a scatter chart showing the different ideas themes which are emergent in the workshop.

There are three different CA agents by creativity workshop. Each agent manages one type of interface.

\section{CONCLUSION}

This paper presents the architecture of a multi agent system dedicated to the ideas management during creativity workshops. The system uses the semantic web language and idea ontology to build research and exploit ideas annotations. The next work of this project will be to make the agents pro active, i.e. to allow the agents to inform the different type of users (Creative participants, creativity quizmasters and stakeholders) all along the workshop about the trend of ideas, or if a new idea is similar to another. 


\section{REFERENCES}

[1] S. Dasgupta, Creativity in Invention and Design, Cambridge University Press, 1994

[2] R. Sternberg, Handbook of Creativity, Cambridge University Press, New York, NY, USA, 1999.

[3] C. Mihaly, Creativity: flow and the psychology of discovery and invention, Harper Collins Publishers, New York, NY, USA, 1996.

[4] E. Arias, H. Eden, G. Fischer, A. Gorman, and E. Scharff, "Transcending the individual human mind creating shared understanding through collaborative design," ACM Trans. Comput. Hum. Interact., vol. 7, no. 1, p. 84-113, 2000.

[5] J. William, S. Alan, and S. Marjory, Beyond Productivity : Information, Technology, Innovation, and Creativity, The National Academies Press, 2003

[6] M. Mumford, Handbook of Organizational Creativity, Imprint: Academic Press, 2011.

[7] R. K. Kazanjian and R. Drazin, "Chapter 21 - Organizational Learning, Knowledge Management and Creativity," in M. D. Mumford (ed.), Handbook of Organizational Creativity, Academic Press, San Diego, pp. 547 - 568, 2012.

[8] P. Paulus and H. Coskun, "Group Creativity," in E. in Chief : Mark A. Runco, S. R. Pritzker(eds), Encyclopedia of Creativity (Second Edition), second edition edn, Academic Press, San Diego, pp. 575 - 580 2011.

[9] S. Ben, "Creativity support tools: accelerating discovery and innovation," Commun. ACM, vol. 50, no. 12, pp. 20-32, December, 2007.

[10] Y. Rogers and S. Lindley, "Collaborating around vertical and horizontal large displays: Which way is best," Interacting with Computers, vol. 16, p. 2004, 2004.

[11] A. F. Smeaton, H. Lee, C. Foley, and S. M. Givney, "Collaborative Video Searching on a Tabletop," Multimedia Systems Journal, vol. 12 no. 4, pp. 375-391, 2006.

[12] M. Morris, J. Lombardo, and D. Wigdor, "WeSearch : supporting collaborative search and sensemaking on a tabletop display," in Proc. of the 2010 ACM conference on Computer supported cooperative work, CSCW'10, ACM, New York, NY, USA, p. 401-410, 2010a.

[13] S. Loke and S. Ling, "Analyzing Observable Behaviours of Device Ecology Workflows," in Proc. of the 6th International Conference on Enterprise Information Systems, pp. 78-83, 2004.

[14] K. L. Dugosh and P. B. Paulus, "Cognitive and social comparison processes in brainstorming," Journal of Experimental Social Psychology, vol. 41, no. 3, pp. 313 - 320, 2005.

[15] A. V. Gundy, "101 activities for teaching creativity and problem solving," 2005

[16] S. Isaksen, K. Dorval, and D. Treffin, The Act of Creation, 2012

[17] S. Buisine, G. Besacier, A. Aoussat, and F. Vernier, "How do interactive tabletop systems influence collaboration," Computers in Human Behavior, vol. 28, no. 1, pp. 49 - 59, 2012.

[18] T. Buzan, The mind map book, Penguin Books, 1991.

[19] C. Shen, F. D. Vernier, C. Forlines, and M. Ringel, "DiamondSpin : an extensible toolkit for around-the-table interaction," in Proc. of the SIGCHI Conference on Human Factors in Computing Systems, CHI '04, ACM, New York, NY, USA, pp. 167-174, 2004.

[20] M. Wooldridge and J. Kinny, "The Gaia Methodology for Agent-Oriented Analysis and Design," Autonomous Agents and Multi-Agent Systems, 2000, vol. 3, no. 3, pp. 285-312.

[21] D. Monticolo, I. Lahoud, and E. Bonjour, "SemKnow: A Multi-Agent Platform to manage distributed knowledge by using ontologies," in Proc. the 2012 IAENG International Conference on Artificial Intelligence and Applications (ICAIA 2012) Hong Kong, 14-16 March, 2012

[22] D. H. Roth, "Agents in Annotated Worlds," in Autonomous Agents, ACM, 1998, pp. 173-180

[23] B. L. Tim, J. Hendler, and O. Lassila, "The semantic web," Scientific American, vol. 284, no. 5, 2001, pp. 28-37.
[24] C. Riedl, S. Wagner, and J. Leimeister, Exploring Large Collections of Ideas in Collaborative Settings through Visualization, 2010.

[25] A. Westerski and C. A. Iglesias, "The road from community ideas to organisational innovation," A life cycle survey of idea management systems, vol. 7, no. 4, pp. 493-506, 2011.

[26] M. L. Deborah and F. V. Harmelen. "OWL web ontology language overview," W3C recommendation, 2004.

[27] S. Savarimuthu, M. Purvis, and M. Purvis, "Tag-based Model for Knowledge Sharing in Agent Society," in the 8th International Conference on Autonomous Agents and Multiagent Systems, 10-15 May, 2009, Budapest, Hungary

[28] G. Fabien, L. Berthelot, and R. D. Kuntz. "A multi-agent platform for a corporate semantic web," in Proc. of the first international joint conference on Autonomous agents and multiagent systems, 2002.

[29] M. Klusch, "Intelligent Information Agents: Agent-based Information Discovery and Management in the Internet," Springer, 1999.

[30] Ferber, Gutknecht, "A meta-model for the analysis and design of organizations in multi-agent systems," IEEE Computer Society, 3rd ICMAS, pp. 128-135, 1998.

[31] O. Daniel and V. Dignum. "OperettA: a prototype tool for the design, analysis and development of multi-agent organizations," in Proc. of the 7th international joint conference on Autonomous agents and multiagent systems: demo papers, International Foundation for Autonomous Agents and Multiagent Systems, 2008.

[32] B. Paolo et al., "Tropos: An agent-oriented software development methodology," Autonomous Agents and Multi-Agent Systems 8.3, pp. 203-236, 2004.

[33] D. Monticolo, I. Lahoud, and E. Bonjour. "Distributed knowledge extracted by a MAS using ontology alignment methods." in Proc. of 2012 International Conference on Computer \& Information Science (ICCIS), vol. 1. 2012.

[34] P. H. Eric and A. Seaborne. "SPARQL query language for RDF," W3C recommendation, vol. 15, 2008.

[35] R. Rada, H. Mili, E. Bicknell, and M. Blettner, "Development and Application of a Metric on Semantic Nets," IEEE Transaction on Systems, Man, and Cybernetics, vol. 19, no. 1, pp. 17-30, 1989.

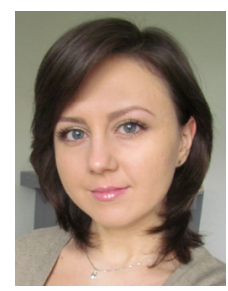

Adriana Simona Mihaita is currently a full time teaching assistant and researcher at the ERPI Laboratory from the University of Lorraine, France. She obtained the $\mathrm{PhD}$ degree in Automatic Control in 2012, from the laboratory Gipsa-lab belonging to the Polytechnic Institute of Grenoble, France. The $\mathrm{PhD}$ thesis presented "A probabilistically approach for applying the event-based control in stochastic switching systems". Her main research interests are related to the hybrid systems, event-based control, Markov Chains, innovation and computational creativity, as well as the flow management.

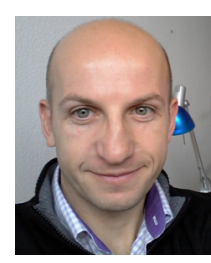

Davy Monticolo is an associate professor in informatics and computer science in the ERPI laboratory of the University of Lorraine, France. He has a Ph.D. in Informatics and Computer Science. His Research interests include: Knowledge Engineering and Modelling, Semantic Web, Ontologies, Web Services and Multi-Agents Systems. His main domain of application is knowledge engineering dedicated to innovation projects and knowledge management in general.

$\mathrm{He}$ is the chair of the IEEE KARE (Knowledge Acquisition Reuse and Evaluation) international workshop. He is also in the board of the AFIA (French Research Group of Artificial Intelligence). 